\title{
THE AORTIC ARCH DERIVATIVES IN THE HUMAN ADULT
}

\author{
ALEXANDER BARRY
}

Department of Anatomy, University of Michigan Medical School

SIX FIGURES

The development and fate of the aortic arch complex has been studied and taught for many years. The work of Congdon ('22), Poynter ('16) and others has left but few gaps in our understanding of the morphological development of this system. Nevertheless, most presentations of both the normal and abnormal development of the great vessels leaving the heart are still based on the summarizing diagram of Rathke (1858), or some modification of it (see fig. 1). This diagram presents succinctly the various components of the aortic arch system, showing in diagrammatic form their original symmetrical pattern, and indicating which components persist and which have ceased to be recognizable in the adult. The chief inadequacy of this type of diagram is that it cannot, by its very nature, show the adult configuration of the great vessels leaving the heart, nor can it show the relatively tremendous alterations in proportion and relationship that have occurred during development.

In addition to these inherent difficulties, the Rathke type of diagram leaves many students confused because it bears a superficial resemblance to the pattern of the arching aorta of the adult. This may account for the confusing half-truths one occasionally encounters, such as the statement that the arch of the aorta of the adult (too frequently miscalled "the aortic arch") represents the left fourth aortic arch of the embryo. 
If one follows the generally accepted facts concerning the development of the aortic arch system, one can subdivide it into more recognizable components than those generally described.
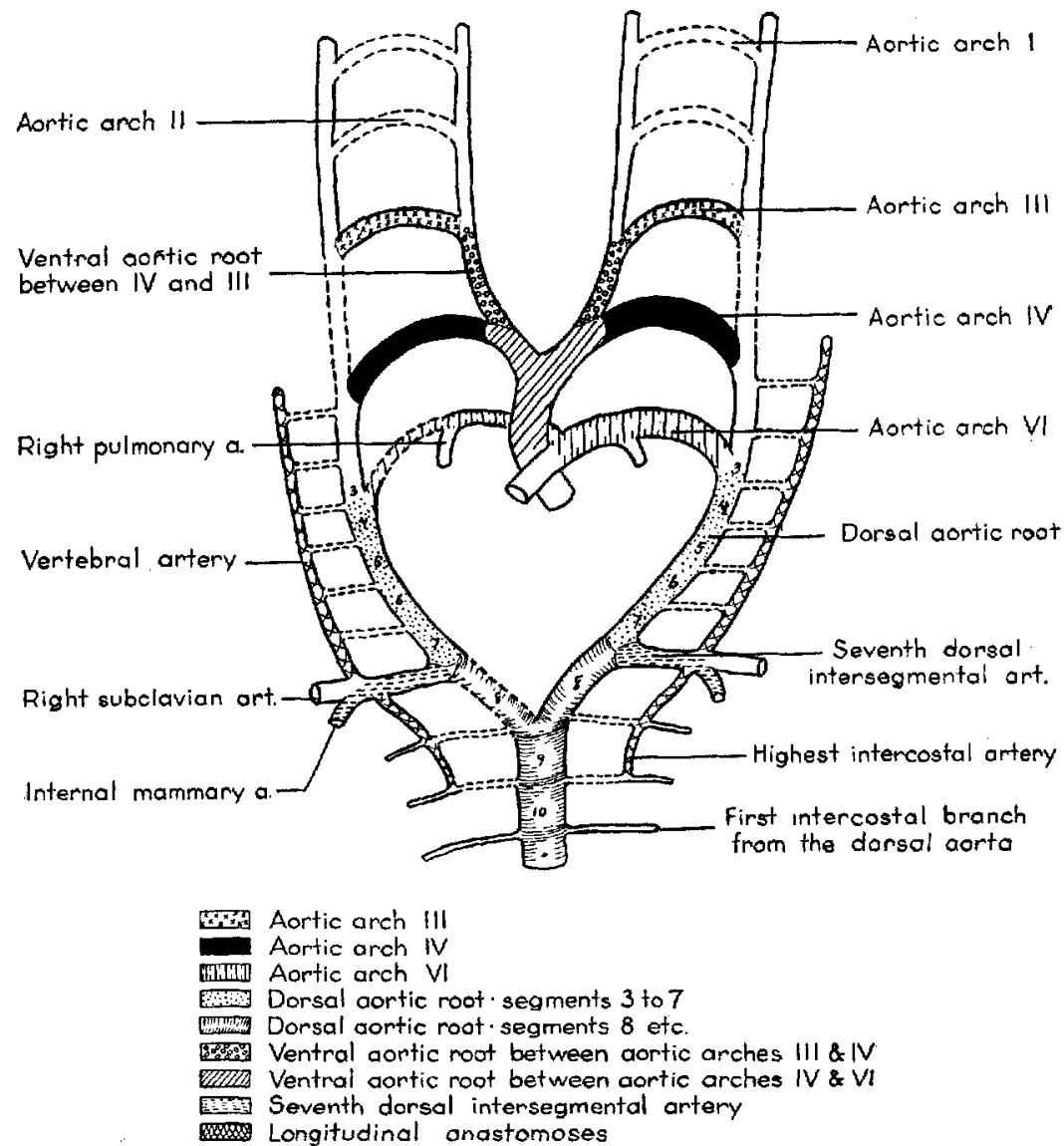

Fig. 1 Schematic diagram indicating the various components of the embryonic aortic arch complex in the human embryo. Those components which do not normally persist in the adult are indicated by broken outlines.

These components can be traced through development to their adult configuration with a reasonable degree of precision and certainty. The present paper presents such an analysis. In the interests of brevity only such phases of development of 
the aortic arch system will be discussed as are necessary to this analysis.

At an early stage of development, the dorsal aortae give off dorsal intersegmental arteries which supply blood to the spinal cord and the developing somites. One can, therefore, regard the dorsal aortae as being subdivided into segments, which fundamentally correspond to the somites. Each of these segments can be identified since it is delimited by the intersegmental arteries, and each can be numbered, by giving it the same number as the intersegmental artery bounding it caudally. As the somites differentiate the axial skeleton with its musculature develops, and effectively anchors the distal ends of the dorsal intersegmental arteries in their original positions with respect to the bodies of the vertebrae and the costal elements. During the second month the heart gradually migrates caudally with respect to the axial skeleton, progressively elongating the arteries which extend between the outlet of the heart and the cervical and upper thoracic levels of the body. $A_{s}$ this process continues, certain segments of the originally symmetrical arterial pattern regress, and others enlarge correspondingly. In addition to this alteration in the length and diameter of the arteries, there are changes in their relationships to one another. One of the most profound of these is the cephalic shift of the origin of the subclavian arteries. Although the embryology of this artery is well known, certain aspects of its development may well be recapitulated here, since its changing relations furnish one of the keys to tracing with precision the adult position of the embryonic components of the arch of the adult aorta.

At the time the anterior appendage bud begins to be of appreciable size it lies opposite the 7th and 8th somites, and normally a branch from the 7 th dorsal intersegmental artery supplies it. During subsequent development this 7 th dorsal intersegmental artery persists and enlarges to constitute the subclavian artery. The first thoracic segment is the 8th in the cervicothoracie series lying immediately caudal to the 7 th cervical. Since the first rib develops in the first thoracic seg- 
ment, the subclavian artery must pass cephalic to it and is thus held relatively fixed with respect to the axial skeleton. As the heart descends in the thorax, the proximal part of the subclavian artery is elongated and at the same time its origin from the aorta moves more and more cephalad. It shifts as if it were being dragged upward by the tension caused by the caudal movement of the heart, although the actual forces involved are probably not as obviously simple.

In the primitive condition each dorsal intersegmental artery divides into a dorsal and a latero-ventral branch; the former chiefly supplying the spinal cord, the vertebral column and its musculature, while the latter follows the lateral body wall, becoming in the thoracic region an intercostal artery. A series of longitudinal anastomoses are secondarily established between the intersegmental arteries. It is not pertinent to attempt to discuss all of these, but two in particular are involved in the present analysis. One of these, the vertebral, is formed as a longitudinal channel dorsal to the costal elements, interconnecting the dorsal rami of the dorsal intersegmental arteries from the first to the 7 th. As the cervical costal elements fuse to their respective vertebrae they enclose the vertebral arteries in vertebral foraminae. Since this anastomosis runs ventral to the 7 th costal element, the vertebral artery does not pass through a vertebral foramen in the 7 th cervical vertebra. After the vertebral artery has been formed, the first 6 dorsal intersegmental arteries running to it become markedly attenuated as the heart descends in the thorax. Gradually, perhaps due to this attenuation, the caliber of these feeder arteries becomes progressively smaller, and eventually they can no longer be identified. This leaves the vertebral artery arising from the subclavian (see fig. 1). The embryonic origin of the subclavian artery from the 7 th dorsal intersegmental artery can be demonstrated even in the adult by two readily seen relationships: its course cephalic to the first rib, and the passage of the vertebral artery through the vertebral foramen of the 6 th cervical vertebra. 
In the thoracic region the ventrolateral rami of the dorsal intersegmental arteries course in the intercostal spaces as the intercostal arteries. Originally all of these vessels arise directly from the dorsal aorta. At about the time of the formation of the vertebral artery, another longitudinal anastomosis forms, interconnecting the ventrolateral rami of dorsal intersegmental arteries 7, 8, and 9. This is a precostal anastomosis, passing ventral to the ribs. The first and second intercostal arteries lose their connection with the dorsal aorta, and are supplied with blood from the subclavian by this new channel which is called the highest intercostal branch of the costo-cervical trunk. The intercostal artery to the third interspace normally remains as a branch from the dorsal aorta, and thus represents the 10th dorsal intersegmental artery (fig. 1).

The fusion of the originally paired dorsal aortae extends cephalically to about the 9th segment. ${ }^{1}$ This is clearly seen at the stage of development reached by an embryo of about $15 \mathrm{~mm}$ crown-rump length (see fig. 2). At this stage one sces another striking result of the relative caudal descent of the heart. The right subclavian artery is held at a relatively high position as it passes over the first rib. This channel, at its origin from the innominate artery is formed from the right 4th aortic arch and that part of the right dorsal aortic root between the 4th aortic arch and the 7 th dorsal intersegmental artery (the third to the 7th segments inclusive). It can be seen from figure 2 that the portion of the right dorsal aortic root between the 7 th dorsal intersegmental artery and the point of union of the paired dorsal aortic roots (segments 8 , and occasionally 9) is markedly attenuated. This narrowed channel will continue to degenerate and can no longer be found in embryos older than $20 \mathrm{~mm}$ erown-rump length.

From such criteria as have been discussed above, figures 3 and 4 were drawn, showing the positions in the adult of the

\footnotetext{
1 The first 8 segments of the primitive paired dorsal aortae do not normally fuse, and for this reason will be designated the dorsal aortic roots, to distinguish them from the more caudal segments of the dorsal aorta which, after the stage of about $15 \mathrm{~mm}$ crown-lump length, represent a fusion of originally paired vessels.
} 
various components of the primitive symmetrical aortic arch system of the embryo. If we recognize the first intercostal artery from the descending aorta as representing the 10th dorsal intersegmental artery, and the subclavian as representing the 7 th, then that part of the greater curvature of the descending aorta lying between them represents the original 8 th, 9th, and 10th aortic segments. The fate of the higher

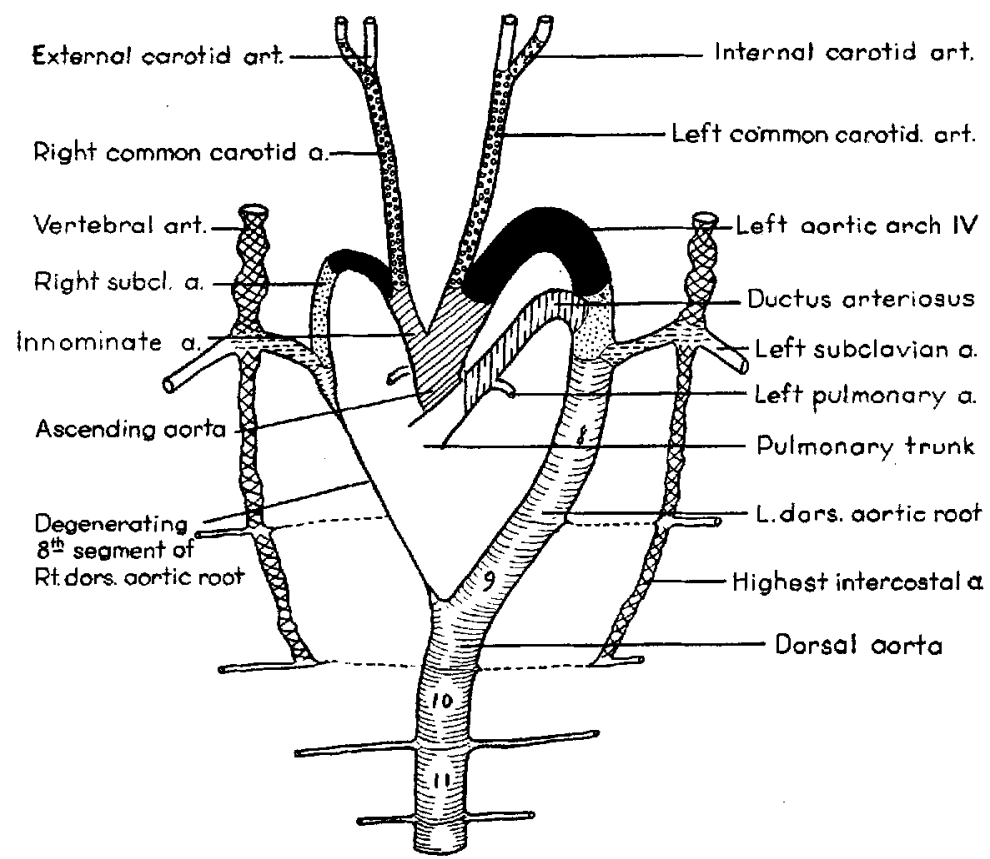

Fig. 2 Diagrammatic ventral view of the aortic arch complex as it appears in a human embryo of $15 \mathrm{~mm}$ erown-rump length. The various components are indicated by the same scheme of symbols as was used in figure 1 .

segments of the left dorsal aortic root is at first glance confusing, since they have undergone extreme compression. As will be seen from figure 1, the part of the arch of the aorta between the left common carotid artery and the left subclavian artery is formed from the left 4 th aortic arch, and the third through the 7th segments of the left dorsal aortic root. The first two segments are not regarded as being represented in 
either the arch of the aorta or in the root of the right subclavian artery for the following reasons. At the stage of development when the 4 th and 6 th aortic arches have been formed, that segment of the dorsal aortic roots between the 4th and third aortic arches shows a narrowing. (This region is sometimes called the carotid duct). As the heart descends
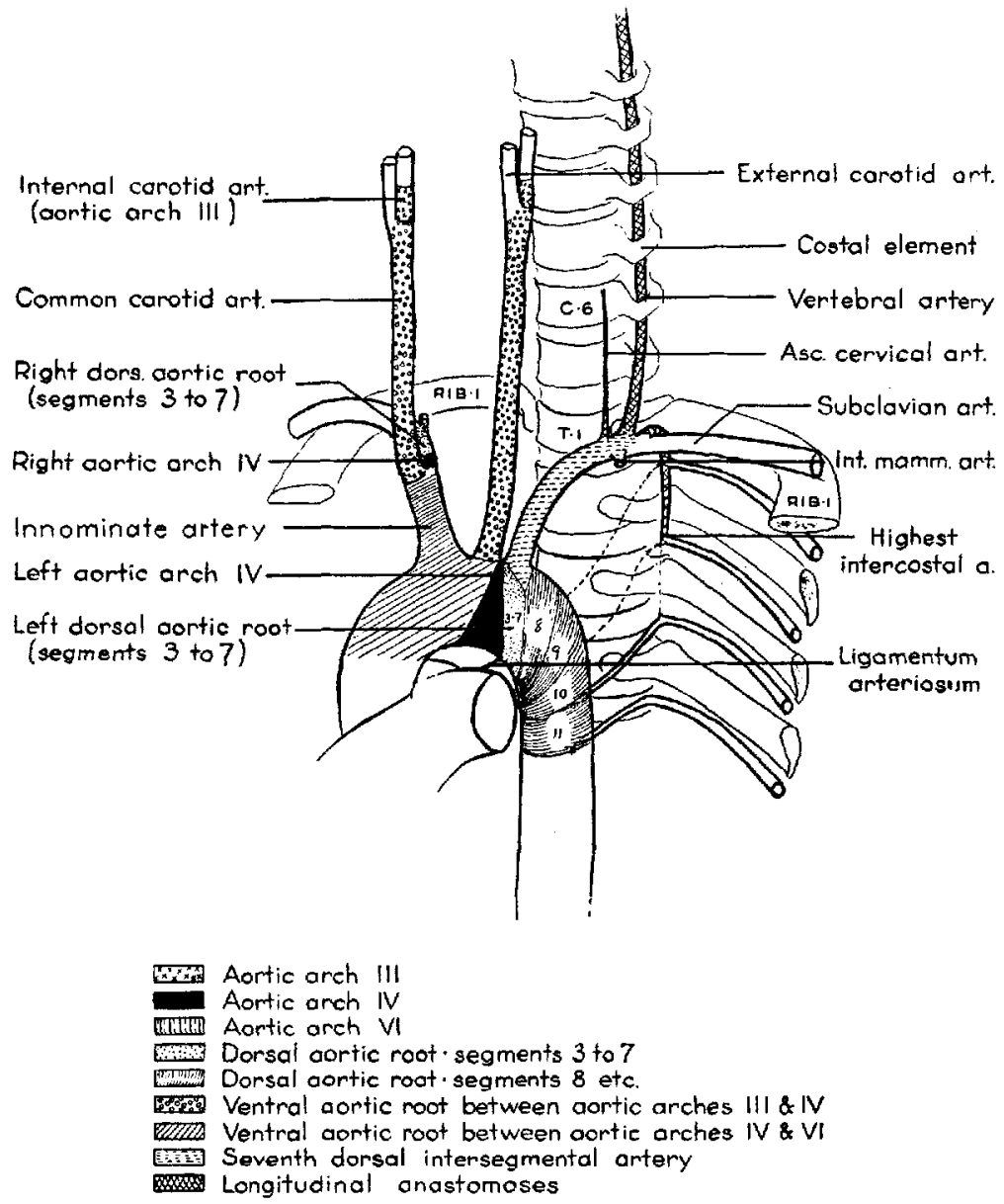

Fig. 3 Jingram of the adult aorta with its main branches as seen from the left ventral aspect. The position of the various components of the embryonic aortic areh complex is indicated in the same schematic manner as was used in the preceding figures. 
in the thorax the origin of the dorsal intersegmental arteries from the dorsal aortic roots move cephalad. By the stage of $14 \mathrm{~mm}$ the carotid duct has narrowed to form a fibrous cord which shortly thereafter disappears. At this stage of development (see Congdon, '22, fig. 13) the third dorsal interseg-

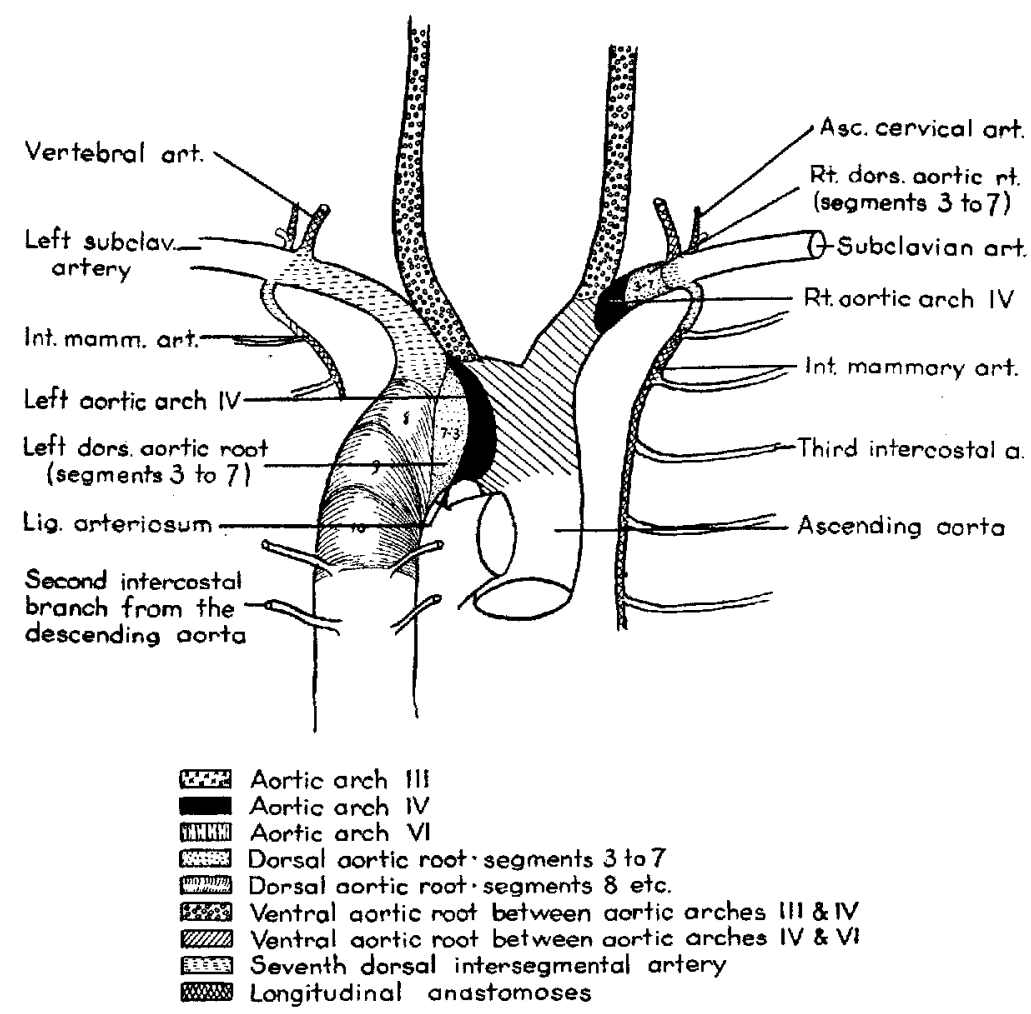

Fig. 4 Diagram of the adult aorta with its main branches as seen from the dorsal aspect. The position of the various components of the embryonic aortic arch complex is indicated in the same manner as in figure 3 .

mental artery has its origin at about the point of junction of the 4th aortic arches and the dorsal aortic roots, while the first and second dorsal intersegmental arteries seem to arise from that part of the dorsal aortic roots that forms the carotid duct and possibly a small part of the internal carotid artery. 
For the sake of diagrammatic simplicity the portion of the arch of the aorta between the common carotid and the subclavian arteries on the left is shown as being composed about equally of the 4th aortic arch and the third to the 7th dorsal aortic root. There seems to be no way to determine the precise degree of participation of these two components, but it would appear that the precise amount each contributes to the arch of the aorta is of no discernible practical importance.

The 6th aortic arch on the left joins the left dorsal aortic root at about the 4 th segment. For this reason the line dividing the 4th aortic arch component of the arch of the aorta from the dorsal aortic root portion at the lesser curvature of the arch of the aorta is drawn to the point where the ductus arteriosus is attached, since the ductus represents the distal portion of the left 6 th aortic arch (fig. 2). There is no way in which the proximal boundary of the part of the aorta arising from the 4th aortic arch can be identified in the adult except at the root of the left common carotid artery, and so the line of demarcation indicated in figures 3 and 4 should be regarded as arbitrary.

That part of the aorta between the ligamentum arteriosum and the first aortic intercostal artery is formed from the 4th to the 10th aortic segments. The oblique lines indicating the positions of these segments are arbitrarily spaced in the figures showing their position in the adult. However, they seem reasonable and gain support from analyses of various anomalous conditions, such as that in which the right subclavian artery arises from the descending aorta. In this case the aberrant artery is derived, as will be discussed in a later paragraph, from the 8th segment of the right dorsal aortic root, and arises from the position indicated for the 8th aortic segment in figures 3 and 4 .

The analysis of the region of the ascending aorta cannot be made with precision in view of the fact that there are no exact landmarks separating its various components. In the young embryo, when the pairs of aortic arches are first forming, the blood is pumped from the beart into an undivided 
truncus arteriosus, which ends in a dilatation which has been called the aortic sac. The aortic arches diverge from this sac. As development progresses the truncus is divided into an aortic and a pulmonary channel. Since there is no clear line of demarcation between the distal end of the truncus and the aortic sac, no attempt has been made to draw such a boundary in any of the diagrams presented. During development the aortic sac extends laterally to the right and left so that the aortic arches on either side appear to arise from horns. In more primitive vertebrates the aortic arches often arise as branches from paired ventral aortae. It would appear that the right and left horns of the aortic sac correspond roughly to these ventral aortae, since they are paired arterial channels carrying blood from the truncus arteriosus to the aortic arches. Indeed some of the sehematic diagrams of the Rathke type show distinct ventral aortic channels, as does figure 1 . This representation admittedly tends to give a false impression as to the length of the channels feeding the various aortic arches. Nevertheless I believe that there is an advantage in retaining in a diagram of this sort some reference to the phylogenetic background of the aortic arch complex. In addition these channels stand functionally in much the same relation to the aortic arches on the afferent side as do the dorsal aortic roots on the efferent side. It is for these reasons that the term ventral aortic root has been used in the figures presented, instead of the term horn of the aortic sac.

The situation on the right side of this aortic arch complex is somewhat different from that on the left, since the disappearance of the 8th segment of the right dorsal aortic root seems to have relieved the tension on the higher segments of this root, allowing them to assume the nearly horizontal position depicted in figures 3 and 4. As is there shown, the root of the right subclavian artery is formed from the right 4th aortic arch. The next part is from the third to the 7th segments of the right dorsal aortic root, while the distal portion is formed from the 7th dorsal intersegmental artery and its branch to the arm. It is possible to estimate the extent of 
these several components in the adult if one uses the vertebral artery as a guide. The proximal portion of the vertebral, it will be recalled, was formed as a longitudinal branch from the 7 th dorsal intersegmental artery, and therefore the right 4th aortic arch and the third to the 7 th segments of the right dorsal aortic root together make up the relatively short length of the adult subclavian artery between its origin from the innominate artery and the point where it gives off the vertebral.

\section{DISCUSSION}

It is hoped that the diagrams presented may serve as a steppingstone toward a better understanding of variations of pattern of the great vessels leaving the heart. It should not be necessary to emphasize that the first step in investigating. the cause of any malformation is the estimating of the probable time and place of its initial departure from normal, for such information at least sheds light on the time and place where the unknown etiological factor or factors were effective.

To ascertain the time of departure from normal in the case of any malformation of the aortic arch complex, it is evident that one must be able to visualize the sequence of morphological relationships of the abnormal segment of the vascular pattern throughout the entire development of the individual. This can be done only as accurately as one can determine the embryological origin of the abnormal segment as it appears in the adult.

For example, one of the relatively common anomalies involving the aortic arch complex is that in which the right subclavian artery originates from the descending aorta instead of from the innominate artery as is usual. Figure 5 shows a drawing of a typical example of this condition. The position of the abnormal origin of the right subclavian artery with relation to the origin of the left subclavian artery, makes it clear that in this condition the 8th segment of the right dorsal aortic root has persisted, forming the proximal part of the right subclavian artery. In most cases of this type one 
can be sure that the distal part of the right subclavian artery was formed as usual from the right 7 th dorsal intersegmental artery, since, as was discussed above, its vertebral branch passes through a vertebral foramen in the 6 th cervical vertebra. If this is the case, then the right 4 th aortic arch and/or the third to the 7 th segments of the right dorsal aortic root underwent regression.
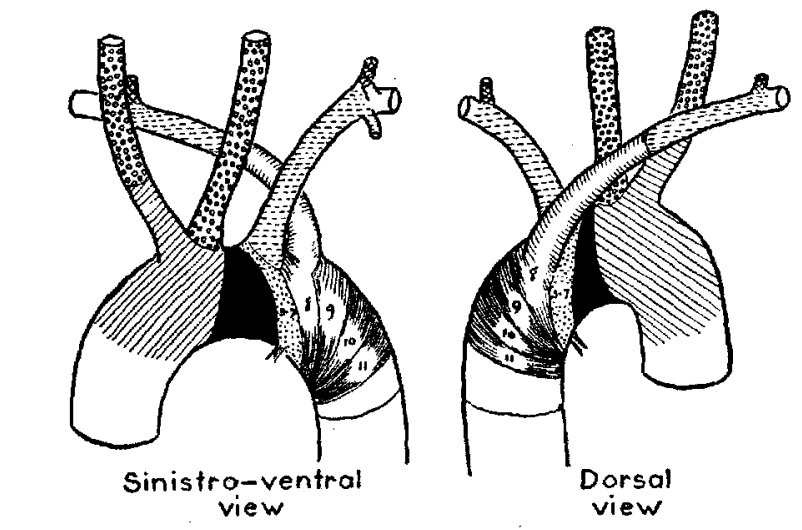

Right subclavian artery arising from descending aorta

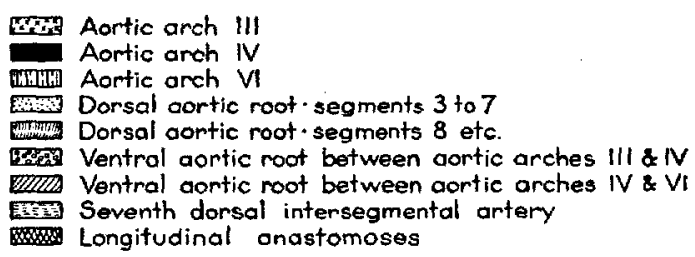

Fig. 5 Two views of the aorta and its major branches as seen in a case in which the right subelavian artery originated from the arta. The position of the various components of the embryonic aortic arch system is indicated as in the preceding figures.

If the 4 th aortic arch on the right and the proximal segments of the right dorsal aortic roots had persisted as well as the 8th segment of the dorsal aortic root on the right, then the trachea and the oesophagus would have been enclosed in a vascular ring, a condition known as a "double aortic arch." In this anomaly all of the components of the embryonic aortic 
arch complex have persisted with the exception of the first two pairs of aortic arches, the distal part of the right 6 th aortic arch and the carotid duets. This type of anomaly, since it represents nearly a complete persistence in the adult of the symmetrical aortic arch system of the embryo, was chosen by Edwards ('48) as the basis for a system of classification of anomalies of the great vessels leaving the heart. Kirklin and Clagett, in 1950, suggested modifying this system by taking as a basis of classification, the extremely rare condition in which the right as well as the left 6 th aortic arches remain identifiable. This modification is well founded, since it does represent as nearly a complete persistence of the embryonic segments as has yet been reported.

It is perhaps unfortunate that the conditions in which a vascular ring surrounds the trachea and oesophagus have been called "double aortic arches." This term tends to imply that they represent a condition in which both right and left 4 th aortic arches have persisted. Actually, as is shown in figure 6, the great artery passing dorsal to the oesophagus and trachea represents a persistent 8 th segment of the right dorsal aortic root.

In figure $6 \mathrm{~B}$ is represented the condition of a double aortic arch with atresia between the left subclavian and left common carotid arteries. In this case the relations of the atretic segment to the subclavian artery, the common carotid artery and the ligamentum arteriosum makes it inescapable that the defect involved the region of the left 4 th aortic arch. In addition, the pattern differs from the normal in that the 8th segment of the right dorsal aortic root has persisted. There seems to be no way of determining the validity of the tempting hypothesis that the atresia was the primary defect and that the persistence of the right 8th segment of the dorsal aortic root was secondary, maintaining an adequate blood supply to the descending aorta in spite of the atretic part of the arch of the aorta. If the 8th segment of the right dorsal aortic root had regressed normally, then the condition would be typical of that in atresia or coarctation of the aorta, at least with 
respect to the course of blood flow, since the arch of the aorta would be narrowed between the left common carotid and subclavian arteries, and the descending aorta would be dependent on a collateral circulation for its blood supply.

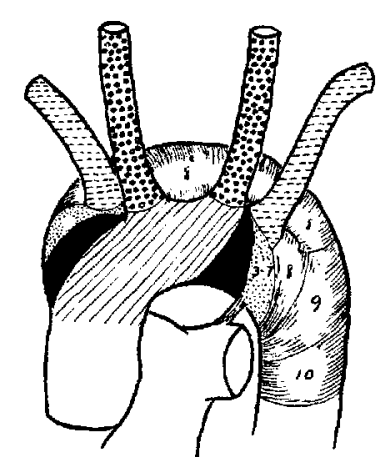

A.

$$
\begin{aligned}
& \text { Double aortic arch } \\
& \text { ventral view }
\end{aligned}
$$

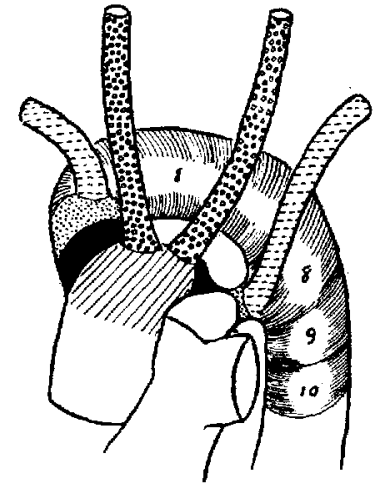

B.

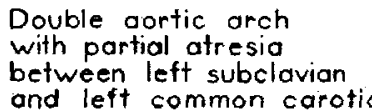

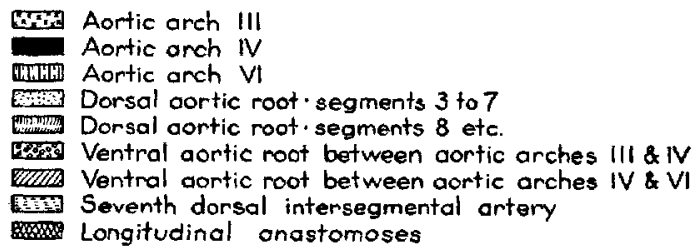

Fig. 6 A. A ventral view of the aorta and its major branclies as seen in a case of double aortic arch.

B. A ventral view of the aorta and its major branches as seen in a case of double aortic arch with extreme constriction of the arch of the aorta between the subclavian and common carotid arteries on the left. In these figures the position of the components of the embryonic aortic arch complex is indicated as in the preceding figures.

If the ligamentum arteriosum were attached to the arch of the aorta proximal to the coarctation, and the left subclavian artery originated distal to it, then it seems probable that the narrowed area was that arising from the upper part of the left dorsal aortic root (see figures 3 and 4 ). Thus we have two cases of coarctation of the aorta which seem nearly 
identical, yet they can be distinguished by the position of the attachment of the ligamentum arteriosum, and at their incipience probably had a considerably different appearance.

It seems reasonable that, whatever the underlying cause may be, a vessel that becomes stenosed or atretic is probably involved in a more or less annular fashion. If this is true, then one might reason that in the case in which there is a coarctation of the arch of the aorta distal to the attachment of the ligamentum arteriosum and proximal to the origin of the left subclavian artery, the narrowing had developed at a time before the third to the 7 th segments of the left dorsal aortic root had been dragged into the oblique position they occupy in the adult. This would mean that the coarctation developed before the origin of the left subclavian artery had moved to a position cephalic to the attachment of the ductus arteriosus, making it probable that the abnormality had its incipience prior to the stage of $20 \mathrm{~mm}$ crown-rump length.

In contrast to the condition just discussed is the type of coarctation in which the constriction is caudal to the left subclavian artery and cephalic to the attachment of the ligamentum arteriosum to the descending aorta. When it appears in this location, the coarctation bears no direct relationship to the developmental pattern, since it cuts across a part of the aorta that is derived from several embryonic segments (see figs. 3 and 4). It seems probable, therefore, that a coarctation in this location developed later than a coarctation located cephalic to the left subclavian artery and distal to the attachment of the ligamentum arteriosum. This conclusion is based on the fact that it would seem to have developed at a stage after the origin of the left subclavian artery had attained its adult position cephalic to the attachment of the ductus arteriosus with the aorta, which would be after the $25 \mathrm{~mm}$ stage.

An additional phase of the investigation of multiple anomalies of the vascular system must deal with determining which defect is primary and which is secondary. In some cases this seems quite obvious, as in the condition of coarctation of the 
aorta with an enlarged collateral circulation. Here it seems inescapable that the coarctation is the primary defect, and that the resulting abnormal flow and pressure pattern lead to the increased diameter and tortuosity of the collateral channels that bridge the coarctation. Such analyses, however, are not always as self-evident. At all stages of development the aortic arch complex presents an intricate arrangement of branching vessels of varying calibers. As blood is pumped through this system there is developed an exceedingly complex haemodynamic pattern of varying blood streams and velocities. It seems probable that here, as in many other regions, the haemodynamic pattern in its turn puts stresses on the segments of the arterial vessels in such a manner as to determine, at least in part, their subsequent developmental configuration. At the present time we can make only the most tentative and elementary analyses of such relationships. In spite of the inadequacy of our understanding of the haemo. dynamic factors, it is still imperative that we have as precise as possible a knowledge of the vascular pattern of an anomaly in all its changes, from incipience to adult pattern.

The sort of analysis presented in figures 3 and 4 can also serve as the basis for the classification of anomalies of the great vessels leaving the heart. Frankly, I hold no particular brief for the value of any system of classification as an end in itself. Nevertheless, if anomalies are classified in a manner that gives hope of leading to a better understanding of their etiology, then it may be contended that such a system has certain fundamental advantages over one based merely on superficial resemblances of physiological or anatomical conditions in the adult. For example, one can devise a classification based on the embryonic aortic arch segments as presented in this paper. It is possible, with a reasonable degree of certainty, to visualize the adult pattern resulting from the regression of any segment or combination of the segments shown in figure 1. Since there are many such segments, the number of theoretically possible anomalies is enormons. 
However, it is obvious that many such patterns would result in a condition which would not be compatible with continued life for the embryo. The remaining possible viable conditions would fall into a relationship based on the embryonic segments affected. A large number of these would be found to have been reported already from observed cases, and, like the periodic table of the chemist, the classification would have a place for as yet unreported types of anomalies. It should be mentioned that such a system would not account for those abnormalities which arise from the development of large arterial channels in regions where such do not normally occur in the young embryo.

It would seems to be a fruitful step to correlate the various possible viable anomalies in this region with their incidence in the population. From such data one could quantitatively estimate the probability of encountering a given anomaly. Of even greater possible value would be that one might learn whether or not there is, at any stage of development, a critical region in the embryo where an unusually high proportion of anomalies arise. This seems not improbable, particularly since it is generally recognized that there are critical times during the embryonic growth of the individual when it seems that development is particularly prone to be distorted by various environmental factors.

\section{SUMMARY}

The caudal migration of the heart and the differential growth of its associated vessels tend to make it difficult to analyse their adult configuration in terms of embryological derivation. A series of diagrams is presented to show the positions of the various recognizable components of the embry. onic aortic arch system as they have come to lie in the adult. A few abnormal configurations have been analysed for comparison with the normal. It is hoped that these diagrams, keyed as they are to segmental levels, will aid in describing. anomalies of the arch of the aorta and its main branches in terms of their probable developmental origin. 


\section{LITERATURE CITED}

Congdon, E. D. 1922 Transformation of the aortie arch system during the development of the human embryo. Carnegie Contrib. to Emb., 14: $47-110$.

Edwards, J. E. 1948 Anomalies of the derivatives of the aortic areh system. M. Clin. North America, 32: 925-949.

Kirklis, J. W., AND O. T. ClaGeit 1950 Vascular "rings" producing respiratory obstruction in infants. Proe. Staff Meet., Mayo Clin., in press.

Poynter, C. W. M. 1916 Arterial anomalies pertaining to the aortie arches and the branches arising from them. University Studies of the University of Nebraska, Lincoln, $16: 229-345$.

RATKKF, H. 1858 Bemerkungen über die Entstehung der bei manchen Vögeln und den Krokodilen verkommenden unpaaren gemeinschaftliehen Carotis. Arch. Anat. u. Physiol. 